\title{
Cardiac tamponade in a patient with severe dengue fever
}

\author{
Ana Isabel Vieira Fernandes ${ }^{[1],[2], ~ C i r o ~ L e i t e ~ M e n d e s ~}{ }^{[3]}$, Raíssa Holmes Simões ${ }^{[1]}$, \\ Ana Elisa Vieira Fernandes Silva ${ }^{[1]}$, Clarissa Barros Madruga ${ }^{[1]}$, Carlos Alexandre Antunes de Brito ${ }^{[4]}$, \\ Lucio Roberto Castellano ${ }^{[2]}$ and Marli Tenório Cordeiro ${ }^{[5]}$
}

\begin{abstract}
[1]. Serviço de Doenças Infecciosas e Parasitárias, Hospital Universitário Lauro Wanderley, Universidade Federal da Paraíba, João Pessoa, PB, Brasil.
[2]. Grupo de Estudos e Pesquisas em Imunologia Humana, Escola Técnica de Saúde, Universidade Federal da Paraíba, João Pessoa, PB, Brasil.

[3]. Unidade de Terapia Intensiva, Hospital Universitário Lauro Wanderley, Universidade Federal da Paraíba, João Pessoa, PB, Brasil.

[4]. Departamento de Medicina Clínica, Universidade Federal de Pernambuco, Recife, Brasil.

[5]. Laboratório de Virologia e Terapia Experimental, Centro de Pesquisas Aggeu Magalhães, Fundação Oswaldo Cruz, Recife, PE, Brasil.
\end{abstract}

\begin{abstract}
A 26-year-old postpartum female presented with symptoms characteristic of dengue fever on the $16^{\text {th }}$ day of puerperium. On the third day of the illness, the patient presented a clinical picture consistent with shock. Tests determined primary infection with dengue virus serotype 2. Cardiac tamponade was confirmed by echocardiography. This rare manifestation is described in a patient without any associated comorbidity.
\end{abstract}

Keywords: Dengue. Severe dengue. Cardiac tamponade.

\section{INTRODUCTION}

Severe manifestations of dengue infections are associated with several complications, including massive bleeding, respiratory failure, liver failure, encephalopathy, myocardial, and other organ dysfunctions. In the last decade, atypical forms of dengue have been described, such as encephalopathy, liver disorders, including acute liver failure, and heart problems ${ }^{1,2}$.

Cardiac involvement in dengue is often limited and benign, but can lead to dengue shock syndrome, which can present as transient myocardial dysfunction ${ }^{3,4}$. In one fatal case of dengueassociated myocarditis, large areas of muscular necrosis and intense interstitial edema were directly associated with dengue virus on the cardiac muscle ${ }^{5}$. When diagnosed and treated early, myocarditis can resolve without sequelae ${ }^{6}$. However, the clinical manifestation of myocarditis in conjunction with persistent hypotension, despite adequate fluid resuscitation, may lead to more aggressive fluid resuscitation, resulting in large pleural effusions and respiratory failure ${ }^{7}$.

Ultrasonography is useful in the prediction of dengue severity, including the identification of early signs of plasma leakage, such as pleural effusion, ascites, perirenal fluid, subcapsular hepatic collection, and pericardial effusion ${ }^{8}$. In severe cases, infection may be associated with hemodynamic consequences leading to refractory shock ${ }^{9,10}$. Pericarditis in

Corresponding author: Profa. Ana Isabel Vieira Fernandes.

e-mail: anaisabelvf@gmail.com

Received 14 September 2016

Accepted 9 August 2017 patients with dengue is extremely rare and usually associated with an extension of a myocardial lesion, rather than a direct deposit of immune complexes ${ }^{6,11}$. Small pericardial and pleural effusions associated with severe plasma leakage have been described in patients with dengue hemorrhagic fever (DHF), but cardiac tamponade is an extremely rare in patients with dengue. We report a case of cardiac tamponade in a postpartum woman with primary dengue virus serotype-2 (DENV-2) in Brazil. This is the second case of cardiac tamponade in a patient with severe dengue fever reported in literature and the first well-documented case in a patient without any associated comorbidity.

\section{CASE REPORT}

A 26-year-old African American woman who underwent a cesarean section presented with high fever, frontal headache, myalgia, and retro-orbital and bone pain on the $16^{\text {th }}$ day postpartum. The patient's pregnancy progressed without complications, and she reported no comorbidities. The patient was admitted to the emergency room of a private hospital in João Pessoa, Paraíba State, Brazil. The patient received hydration, and initial laboratory examination identified hemoconcentration, leukopenia, and mild thrombocytopenia (Table 1). The woman's condition improved, and she was discharged with symptom severity guidance. Two days later, the patient returned to the hospital with dizziness, cold sweats, a prostration-related fainting episode, lid edema, nausea, vomiting, abdominal pain, diarrhea, mild uterine bleeding, and exanthema.

Later, on the same day of admission, the patient was alert and oriented upon physical examination, but presented with respiratory distress. The patient was dehydrated and 
TABLE 1

Evolution of laboratory tests for the patient with dengue following hospital admission.

\begin{tabular}{|c|c|c|c|c|c|c|c|c|c|c|c|c|}
\hline \multirow[t]{2}{*}{ Exam } & \multirow[t]{2}{*}{$\mathbf{R V}$} & \multirow[t]{2}{*}{ BA } & \multirow[t]{2}{*}{ At admission } & \multicolumn{9}{|c|}{ Days after admission } \\
\hline & & & & 1 & 3 & 4 & 5 & 6 & 7 & 8 & 10 & 14 \\
\hline \multicolumn{13}{|l|}{$\mathrm{CBC}$} \\
\hline Hemoglobin & $12-16 \mathrm{~g} / \mathrm{dL}$ & 15.6 & 8.4 & 15.7 & 12.2 & 10.6 & 11 & 11.2 & 10.5 & 10.6 & 12.3 & 10.3 \\
\hline Hematocrit & $4-5.2$ millions $/ \mathrm{mm}^{3}$ & 44.8 & 25.1 & 46.3 & 36.6 & 31.9 & 33.8 & 34,2 & 32 & 32,6 & 38,1 & 31 \\
\hline Leukocyte $(\mathrm{x} 1,000)$ & $4.2-11.0 / \mathrm{mm}^{3}$ & 3.7 & 4.4 & 10.8 & 8.0 & 9.0 & 10.4 & 11.3 & 7.9 & 8.7 & 10.8 & 7.7 \\
\hline Neutrophils & $40-70 \%$ & 70 & 65 & 70 & 83 & 80 & 82 & 78 & 79 & 76 & 69 & 69 \\
\hline Rods & $0-5 \%$ & 0 & 0 & 0 & 0 & 0 & 0 & 0 & 0 & 0 & 0 & 0 \\
\hline Platelets (x1,000) & $150-450$ thousand $/ \mathrm{mm}^{3}$ & 169 & 115 & 133 & 170 & 203 & 316 & 304 & 287 & 239 & 324 & 323 \\
\hline \multicolumn{13}{|l|}{ Coagulation } \\
\hline APTT & $10-14$ & 10.2 & 10.2 & 10.2 & 10.2 & 10.4 & - & - & - & 11,3 & - & \\
\hline PTT & $100 \%$ & 100 & 100 & 100 & 100 & 97 & - & - & - & 84 & - & \\
\hline \multicolumn{13}{|l|}{ Biochemistry } \\
\hline AST & $11-39 \mathrm{U} / \mathrm{L}$ & 29 & 38 & - & - & 261 & - & - & - & - & 83 & \\
\hline ALT & $11-45 \mathrm{U} / \mathrm{L}$ & 35 & 26 & - & - & 143 & - & - & - & - & 162 & \\
\hline Albumin & $3.5-5.5 \mathrm{~g} / \mathrm{dL}$ & & 1 & - & - & 1.8 & - & - & - & - & 5.2 & \\
\hline C-reactive protein & $<5 \mathrm{mg} / \mathrm{L}$ & & 6.4 & - & - & - & - & - & - & - & 87.2 & \\
\hline
\end{tabular}

RV: reference value; BA: before admission; CBC: cell blood count; APTT: activated partial thromboplastin time; PTT: prothrombin time test; AST: aspartate transaminase; ALT: alanine transaminase.

had an axillary temperature of $38.7^{\circ} \mathrm{C}$, a heart rate of $130 \mathrm{bpm}$, a thready pulse, cold sweats, a blood pressure of $80 / 40 \mathrm{mmHg}$, and evidence of a positive tourniquet test. The patient also exhibited a diminished vesicular murmur at the base of the right lung, hypophonetic heart sounds, and jugular venous distention. Ancillary exams upon admission (the third day of illness) showed decreased platelet numbers and hemoconcentration, and serological tests were positive for immunoglobulin M (IgM), but negative for dengue virus immunoglobulin $\mathrm{G}(\mathrm{IgG})$, and positive for the dengue virus non-structural protein 1 (NS1) antigen. Reverse transcription polymerase chain reaction (RT-PCR) results were positive for the DENV-2 serotype.

The patient was transferred to the Intensive Care Unit (ICU). with a blood pressure of $80 / 40 \mathrm{mmHg}$ and developed metabolic acidosis and persistent arterial hypotension, despite adequate volume expansion. Upon examination, the patient still exhibited hypophonetic heart sounds and jugular venous distention. She was dyspneic and put under alternating noninvasive ventilatory support. Chest radiography and abdominal ultrasound were unremarkable (Figure 1), showing relative hepatomegaly and increased uterine volume, both common during the postpartum period. The patient underwent an echocardiogram, which confirmed the diagnosis of cardiac tamponade (Figure 2). Pericardial fluid was drained and analyzed and was negative for bacterial or fungal structures. However, rare leukocytes were identified after 48 hours of bacterial culture.
The patient developed anasarca with a progressive increase in pleural effusion and ascites. On the third day in the ICU, the patient was administered noradrenaline and $400 \mathrm{mg} / \mathrm{Kg} / \mathrm{day}$ immunoglobulin for three days. On the sixth day, norepinephrine was gradually withdrawn, and the patient developed anxiety and restlessness, requiring oxygen supplementation via a Venturi mask (50\%). A diuretic was initiated. Echocardiography was performed, showing mild pericardial effusion and mitral regurgitation. On the eighth day, the patient showed an improvement in breathing patterns and was discharged from the ICU. In the ward, the patient exhibited an improvement in anxiety symptoms, occasional dry cough, rales in the right lung base, normophonetic heart sounds without jugular venous distention, a heart rate of $80 \mathrm{bpm}$, and a blood pressure of 120/80 mmHg. She exhibited ascites and edema of the lower limbs and genitalia. Chest $\mathrm{x}$-rays showed right inferior lobe atelectasis, small bilateral pleural effusion, and normal heart size. The patient underwent respiratory physiotherapy and was discharged without sequelae.

\section{Ethical considerations}

This work was part of a larger project approved by the UFPB Ethical Committee on Human Research (protocol \#032/2009/ CEP/HULW/UFPB). Written informed consent was obtained from the patient for publication of this case report. 

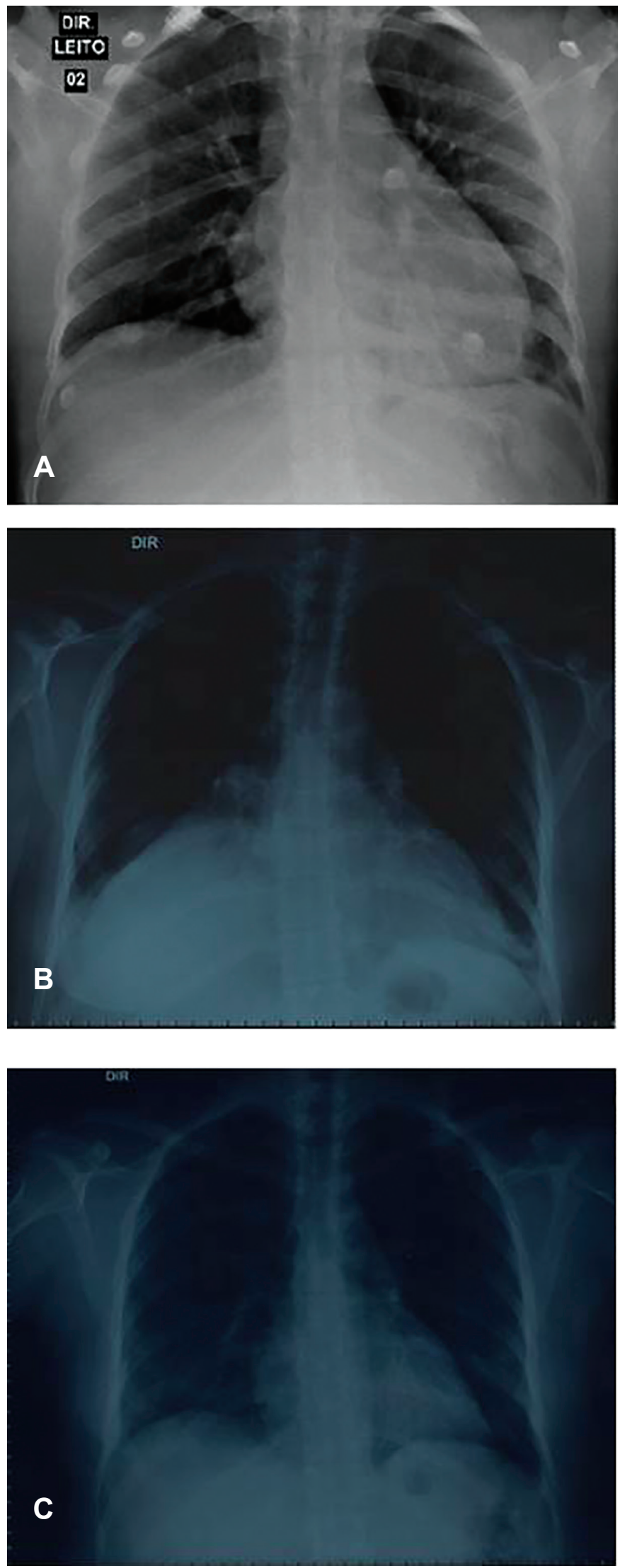

FIGURE 1 - Evolution of the chest radiograph. Increase in heart size and absence of pleural effusion (a). Atelectasis of the lower lobe of the right lung (b). Chest X-ray control to normal discharge from the hospital (c).

\section{DISCUSSION}

This is one of the few cases reported in literature of cardiac tamponade in a patient with dengue fever and the first welldocumented case in Brazil. In addition, this case is clinically important because it was a primary dengue infection of presumably mild intensity. Early in the course of disease, the patient presented with severe persistent vomiting and abdominal pain, requiring fluid resuscitation. On the third day, the patient still had a fever and developed signs of shock, presenting with large pleural effusion and significant vascular endothelium and hypoalbuminemia impairment. During resuscitation, she received a large volume of fluids followed by vasoactive drugs. However, since hypervolemia can cause an increase in effusion levels, the patient's condition was reversed after the shock phase via diuretics and water restriction. In the present case, the pericardial effusion that progressed to cardiac tamponade occurred prior to the other effusions that increased after volume resuscitation.

In a previous case of cardiac tamponade in a female patient with dengue fever, the 59-year-old woman had lupus, which may predispose the person to serositis and stroke ${ }^{9}$. In the present case, puerperium, during which the hypervolemic condition of pregnancy is maintained, may slowed the elimination of liquids observed three weeks after the patient gave birth. There is limited data on the association between DENV infection and cardiac tamponade. Previous reports on adult men based diagnoses on $\operatorname{IgM} / \operatorname{IgG}$ serology, without any confirmatory viral isolation and/or molecular identification of DENV serotypes ${ }^{5,12}$. Further, only one study tested for the NS1 viral protein, demonstrating undetectable circulating levels $\mathrm{s}^{5}$. The present case showed elevated NS1 levels and had positive IgM results, but had negative $\operatorname{IgG}$ results with a confirmatory RT-PCR for the DENV-2 serotype. Immunoglobin serology without any other diagnostic method could present challenges in the determination of the exact role played by the DENV-2 serotype. Previous studies have suggested that positive IgM and IgG results in patients with cardiac tamponade may indicate a DENV secondary infection. In the present case, the patient had negative IgG results, but positive IgM results for DENV, suggesting a primary DENV infection. In comparison with patients with secondary DENV infections, patients with primary DENV infections show fewer or no cardiac complications.

In India, retrospective studies evaluating dengue fever cases among the general population ${ }^{13}$ and adolescents ${ }^{14}$ have reported the occurrence of myocarditis and pleural or pericardial effusion. In the first study ${ }^{13}, 96$ (56.8\%) out of 169 patients did not present positive results for any confirmatory test of DENV infection. For these patients, referred to as the Sero-Negative Probable Dengue Fever group, only epidemiological information about a dengue outbreak in the studied area was provided by the authors. This was a limitation similar to that of the present case. In the second study ${ }^{14}$, DENV infection was described in children, not adults. In literature, differences in the disease course of DENV have been well documented between children and adults, which may make the comparison of the retrospective study to the present case difficult. In addition, both studies lacked confirmatory results to determine the presence of a cardiac tamponade event in their patients. 

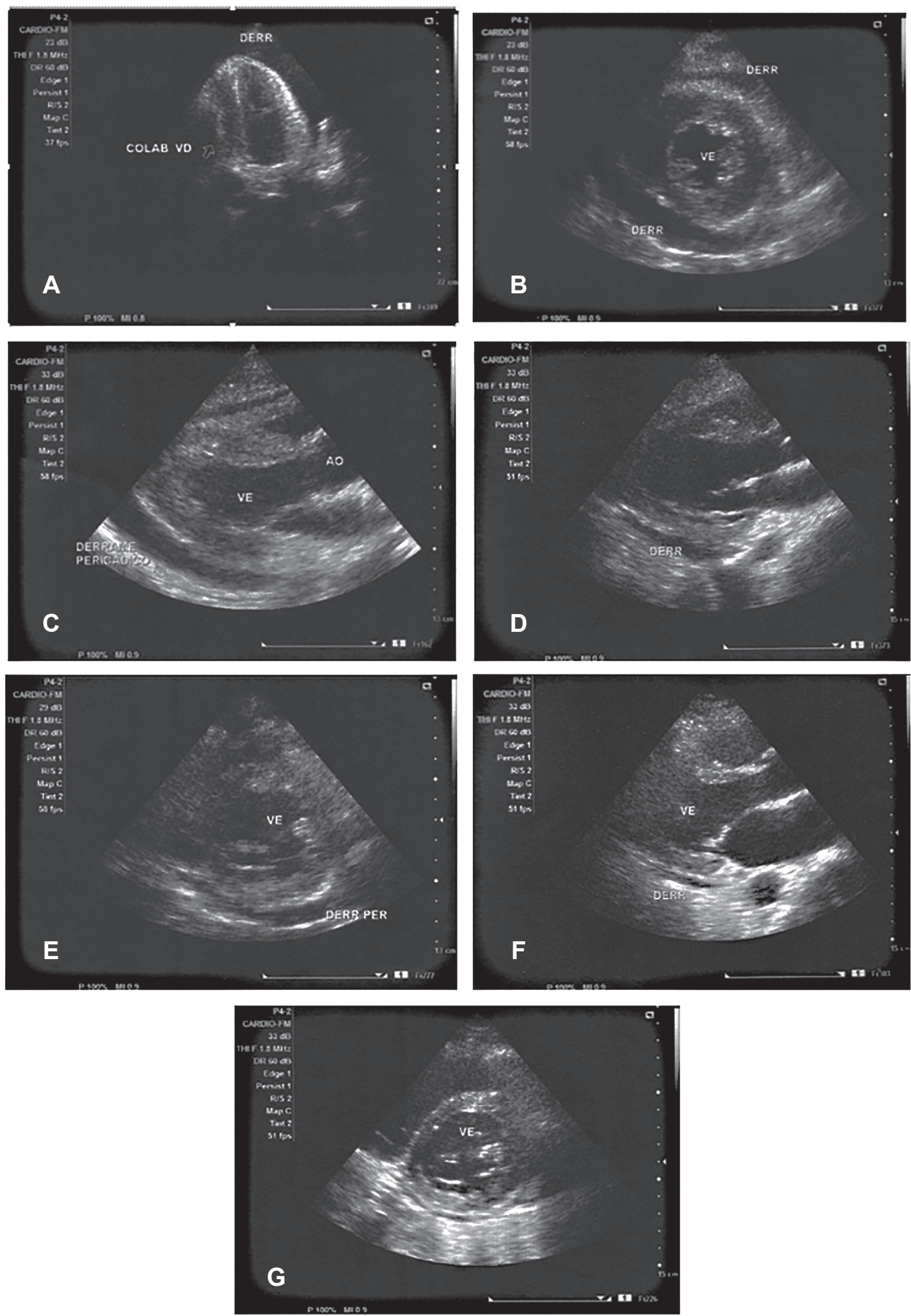

FIGURE 2 - Echocardiogram demonstrating extensive pericardial effusion with cardiac tamponade and right ventricle collapsed (a-c). Control echocardiogram after pericardial drainage. Moderate pericardial effusion without evidence of cardiac tamponade (d-e). Echocardiogram control after four days of pericardial drainage. Small pericardial effusion (f-g). RV: right ventricle. 
RT-PCR identified the serotype as DENV-2. Although other dengue serotypes may be associated with cardiac involvement, DENV-2 is believed to be the main cause of myocardial diseases ${ }^{10}$. Here, no signs of myocarditis were found; however, the association between specific DENV serotypes and cardiac involvement needs to be clarified within a large cohort. The present case opens the discussion of this complication of dengue infection.

Although the patient presented with severe symptoms, she did not fulfill the criteria for DHF because she maintained a platelet count above 100 thousands $/ \mathrm{mm}^{3}$, illustrating a gap in the traditional classification of the World Health Organization ${ }^{15}$. In the revised classification ${ }^{1}$, the case would meet the criteria for severe dengue (SD) because of the development of large effusions in combination with respiratory failure and severe organ dysfunction, exemplified here by cardiac tamponade. This is, however, an extremely rare condition that by itself does not justify a change in disease classification.

Cardiac alterations in patients with dengue may be associated with shock dengue syndrome and should be diagnosed via echocardiography. Progression to cardiac tamponade in patients with dengue is a very rare condition that requires a high degree of suspicion and immediate intervention.

\section{Acknowledgments}

The authors would like to express their gratitude to the patient and to the staff and residents of the Infectious and Parasitic Diseases Service, Hospital Universitário Lauro Wanderley, Universidade Federal da Paraíba, João Pessoa, PB, Brasil.

\section{Financial support}

This work was supported by Conselho Nacional de Desenvolvimento Cientifico e Tecnológico (CNPq), Brazil, grant 576214/2008-6.

\section{Conflict of interest}

The authors declare that there are no conflicts of interest.

\section{REFERENCES}

1. World Health Organization (WHO). Dengue: guidelines for diagnosis, treatment, prevention and control: new edition. France: World Health Organization; 2009. 147p.
2. Weerakoon KG, Kularatne SA, Edussuriya DH, Kodikara SK, Gunatilake LP, Pinto VG, et al. Histopathological diagnosis of myocarditis in a dengue outbreak in Sri Lanka, 2009. BMC Research Notes. 2011;4:268.

3. Gupta VK, Gadpayle A. Subclinical cardiac involvement in dengue haemorrhagic fever. J Indian Acad Clin Med. 2010;11(2):107-11.

4. Miranda CH, Borges Mde C, Schmidt A, Pazin-Filho A, Rossi MA, Ramos SG, et al. A case presentation of a fatal dengue myocarditis showing evidence for dengue virus-induced lesion. Eur eart J Acute Cardiovasc Care. 2013;2(2):127-30.

5. Miranda CH, Borges MC, Matsuno AK, Vilar FC, Gali LG, Volpe GJ, et al. Evaluation of cardiac involvement during dengue viral infection. Clin infect Dis. 2013;57(6):812-9.

6. Wiwanitkit V. Dengue cardiac infection, A Brief Review. Acta Cardiol Sin. 2008;24:226.

7. Pesaro AE, D'Amico E, Aranha LFC. Dengue: cardiac manifestations and implications in antithrombotic treatment. Arq Bras Cardiol. 2007;89(2):e12-5.

8. Oliveira RVB, Rios LTM, Branco MRFC, Braga Jr LL, Nascimento JMS, Silva GF, et al. Valor da ultrassonografia em crianças com suspeita de febre hemorrágica do dengue: revisão da literatura. Radiol Bras. 2010;43(6):401-7.

9. Kumar S, Iuga A, Jean R. Cardiac tamponade in a patient with dengue fever and lupus nephritis: a case report. J Intensive Care Med. 2010;25(3):175-8. Epub 2010/01/22. doi: 10.1177/0885066609358955. PubMed PMID: 20089524.

10. Kularatne SA, Pathirage MM, Kumarasiri PV, Gunasena S, Mahindawanse SI. Cardiac complications of a dengue fever outbreak in Sri Lanka, 2005. Trans R Soc Trop Med Hyg. 2007;101(8):804-8.

11. Nagaratnam N, Siripala K, de Silva N. Arbovirus (dengue type) as a cause of acute myocarditis and pericarditis. Br Heart $\mathrm{J}$. 1973;35(2):204-6.

12. Bendwal S, Malviya K, Jatav OP, Malviya K. Cardiac tamponade presenting as early manifestation in dengue fever. $\mathrm{J}$ Assoc Physicians India. 2014;62(3):257-9.

13. Motla M, Manaktala S, Gupta V, Aggarwal M, Bhoi SK, Aggarwal $\mathrm{P}$, et al. Sonographic evidence of ascites, pleura-pericardial effusion and gallbladder wall edema for dengue fever. Prehosp Disaster Med. 2011;26(5):335-41.

14. Pothapregada S, Kamalakannan B, Thulasingam M. Clinical profile of atypical manifestations of dengue fever. Indian J Pediatr. 2016;83(6):493-9.

15. World Health Organization (WHO). Dengue haemorrhagic fever: diagnosis, treatment, prevention and control. 2nd edition. Geneva: World Health Organization; 1997. 84p. 